\title{
DAVE: Optimizing Wasabi Agriculture Through Automation and Successive Approximation
}

\author{
Jayden Lefebvre ${ }^{1}$
}

${ }^{1}$ Trinity College School, Port Hope, ON L1A 4K7, Canada

\begin{abstract}
$\underline{\text { ABSTRACT }}$
Wasabi agriculture continues to predominantly rely on traditional practices. There currently exists an abundance of botanical literature surrounding the optimization of the wasabi growth environment to increase crop quality; however, this research is detached and independent. Considering the recent rise to prominence of cyber-agriculture technology, its use in further optimizing the wasabi growth environment should be considered. By designing and constructing two wasabi-oriented food computer prototype iterations, uniting and synchronizing the results of existing wasabi optimization research, and growing Wasabia japonica plants inside these food computers, it was found that despite the widely reported difficulty of traditional wasabi farming, the crop is a strong contender for novel cyber-agriculture: the plant tissues showed a 69.3\% increase in flavour compound (allyl isothiocyanate) concentration overall. The plants also exhibited a $74.7 \%$ decrease in overall plant mass, pointing to a well-documented phenomenon dubbed the "dilution effect" present in intensive agriculture. Overall, given future design improvements and more extensive data collection, there exists the possibility to revolutionize wasabi agriculture by engineering cyber-agriculture solutions tailored to wasabi growth.
\end{abstract}

\section{Introduction}

Wasabia japonica, better known as wasabi, is a semi-aquatic plant of the family Cruciferae that has garnered significant culinary interest over the past few decades. Endemic to the mountainous regions of Japan, wasabi is traditionally grown and harvested for its hardy green stalk, called the rhizome [1]. A paste made from the rhizome has been used as a condiment or accompaniment for a variety of traditional Japanese foods for centuries [1], however Wasabi has grown to become revered globally since its introduction to the Western kitchen in the early 20th century [1]. With ever-increasing demand for the irreplaceable ingredient and limited supply due to its notorious difficulty to grow [1], wasabi is one of the most lucrative crops, fetching on average 100USD $/ \mathrm{kg}$ on the market in 1991 with prices continuously increasing [2]. Other nations such as New Zealand [3] and Poland [4] have attempted to replicate the endemic growth environment to take advantage of this market with varying degrees of success; however, the Japanese standard of quality remains unrivalled [1].

\section{Literature Review}

Flavour Chemistry and the Wasabi Market

Through aggregation of numerous informal sources and traditional Japanese knowledge in [1], Washington State University researchers Chadwick et al. confirmed that wasabi is primarily prized for its uniquely pungent flavour and use as a condiment. Two individual papers, [5] and [6], both co-written by Sultana - researcher of Applied Chemistry and Chemical Technology at Dhaka University - and Savage - researcher in the Food Group, Agriculture and Life 
Sciences Division at Lincoln University - support this, and document that the flavour of wasabi can be attributed to the high concentrations of various isothiocyanate compounds ("ITCs"), the most abundant ( $86 \%-92 \%$ of total ITCs) being Allyl Isothiocyanate ("AITC"). Food and Nutritional Sciences researchers Bell et al. at the University of Reading build upon Sultana and Savage's work by investigating the subjective flavor perceptions of each of the compounds individually, and found that AITC is described as "bitter[-tasting], pungent, sulfurous, mustard-like, lachrymose, [and] horseradish-like." As a condiment, "the more pungent [the wasabi] is, the better from a culinary perspective" [7].

Though found to a varying degree throughout the plant, researchers Shin, Ghimeray, and Park from the Department of Bio-health Technology at Kangwon National University found through extraction and gas chromatography-mass spectrometry (GCMS) analysis that AITC is most concentrated in the rhizome [8]. Considering the rhizome is the primary commercial product of the wasabi crop, and that AITC is both the primary flavor compound associated with wasabi quality and the most abundant ITC, both the chemotypic expression of AITC in wasabi and the mass of the rhizome can be used as reliable quantitative indicators of the quality of the wasabi crop, and reflect on the degree of optimization of any particular growth environment.

\section{Environment Variable Optimization}

Given both the cultural and medical importance of wasabi [1], the optimization of individual variables of the wasabi growth environment - with a specific focus on increasing AITC concentration - has been an area of abundant and significant study. For example, Oguni, et al. - all members of the Shikoku Research Institute and/or the Graduate School of Bio-Applications and Systems Engineering at the Tokyo University of Agriculture and Technology - investigated in [9] the impact that "minimum nocturnal ambient air temperature, day length and culture medium temperature [posed on crop] yield ... for the purpose of developing a year round cultivation system for wasabi". The experiment was housed in a greenhouse environment, and researchers were able to control the temperatures via electronic heating and cooling systems. It was found that air temperatures within the range of $12^{\circ} \mathrm{C}-15^{\circ} \mathrm{C}$, as well as root zone temperatures within the range of $10^{\circ} \mathrm{C}-13^{\circ} \mathrm{C}$, were the optimal for wasabi quality.

Artificial fertilizer use in wasabi agriculture is a relatively new phenomenon. Little is currently known about the plant nutrient requirements of wasabi [1], and this particular aspect of the wasabi growth environment is on the cutting edge of wasabi botanical research. The decision of whether or not to use fertilizers at all is still a point of debate between traditional Japanese farmers, modern commercial growers, and researchers worldwide. The team of Sultana et al. from both Lincoln University and the New Zealand Institute for Crop and Food Research performed an experiment in a setup comparable to that of Oguni et al. to evaluate the impact fertilizer type had on AITC concentration. It was discovered that "Fertilisation with ammonium sulphate produced the highest-quality rhizomes (72\% increase in AITC yield) [while] Nitrogen fertiliser alone reduced the AITC yield by up to 15\%" [10]. Craigie, a master's student from Lincoln University, and the team of Martin, Deo, and Depree, researchers at the New Zealand Institute for Crop \& Food Research, performed separate investigations on wasabi fertilization expanding on the research of Sultana et al., and found complementary results using the same kind of greenhouse model. It was found that organic nitrogen fertilizers (i.e. manure) could be effectively replaced by artificial nitrogen fertilizers [11]. In addition, it further confirmed Sultana et al.'s finding that the use of ammonium sulphate as a source of both bioavailable nitrogen and sulfur results in a meaningful increase of AITC concentration, and that fertilization during the latter stages of plant development was optimal [12].

Chadwick et al.'s compiled source [1] contains several ideas on various aspects of wasabi agriculture, including ideal growth medium $\mathrm{pH}$, plant nutrient selection and concentration, and root zone oxygenation, as well as various environment temperatures, which all align with more recent quantitative research.

Given that the quality of plant growth is impacted directly by these variables, they must all be considered in the development of an optimal growth environment. Sultana agrees with this concept, emphasizing that "These results [surrounding plant nutrient program and other variables] should help in formulating guidelines for production of highquality wasabi [crop] containing high levels of AITC" [10]. However, due to the organic nature of traditional growth 
environments, even with human interference and monitoring, there will be uncontrolled and unintended fluctuations in these variables. In terms of mathematically efficient optimization, these variations are not negligible.

\section{Cyber-Agriculture and Hydroponics}

The concept of computer-integrated plant growth systems, or "cyber-agriculture", is a fairly recent one; however, as documented by researchers Tembe, Khan, and Acharekar of St. Xavier's Institute of Engineering, its popularity is limited by frequent closed-source design material and high cost when compared to traditional farming [13]. Fortunately, senior researchers Harper of the MIT Media Lab and Siller of CINEVESTAV Unidad Guadalajara have jointly pioneered the OpenAgriculture Initiative (OpenAG) [14]. OpenAG outlines an open-source design paradigm addressing the possibility for collaboration between the worlds of computation, data science, and agriculture for the implementation of food computers, enclosed microclimates equipped with sensors and actuators optimized for food crop growth. The food computer concept is outlined in such a way that is modular and open source enough to "allow for [food computers] customized in size and design, according to their application". For example, a recent specific instantiation of the OpenAG framework can be seen in the work of Johnson et al. from the MIT Media Lab, the University of Texas at Austin, Cognizant Technology Solutions, and Harvard University to optimise the flavour compound concentrations of Ocimum basilicum, or basil [15]. In this specific instance, a machine learning algorithm was trained to model the relationship between the environment variables (input) and the concentration of specific flavour compounds in the basil plant (output) in order to optimize the growth environment to maximize that concentration. This research was successful, in that over the course of the experiment, it was found that "The highest density of flavor molecules was produced by subjecting the plants to all-day light, which the surrogate optimization approach discovered quickly and reliably". An additional intriguing finding of this paper was the reinforcement of the existence of a "dilution effect'; i.e. a negative correlation between weight and desirable chemical species", meaning that despite the decreased mass of the plant overall, the concentration of the particular compounds in question increased (and vice versa). This experiment and its resulting discussion speak highly of the potential for research in the field of cyber-agriculture.

In addition to the basil-oriented instantiation, the OpenAG framework is well-documented in the "Personal Food Computer" ("PFC") [16], a smaller and more accessible design produced by Ferrer et al. of the MIT Media Lab. The PFC is designed as "a low-cost desktop size platform, which not only targets plant phenology researchers but also hobbyists [and] makers ... The PFC is completely open source [and] can be used in a large spectrum of activities". Ferrer et al.'s paper outlines the bill of materials (BOM) - including sensors, actuators, and construction materials as well as the concept of 'climate recipes' - lists of the specific environment variable values - "for specific organisms where a certain trait (e.g., volume, taste, chemical concentration, etc.) is maximized".

\section{Research Focus}

Despite the abundance of research surrounding the optimization of each variable of the wasabi growth environment independently, the extent to which these components as a collective climate can be induced through computer automation has not yet been explored. Wasabi agriculture still largely hinges on attachment to traditional growing methods [1], and an investigation into the possible application of an OpenAG-observant technology could result in a revolution of thought. This research attempts to address this gap through design and implementation of a PFC analogue tailored to the wasabi growth environment. More specifically, this study aims to investigate the degree of success of OpenAGcompliant devices designed to increase the commercial value of wasabi crops grown therein. 


\section{Overview of Process}

This study used an experimental mixed method to evaluate the efficacy of prototypes developed to approach the optimal wasabi growth environment. Two prototypes were created to test the hypothesis that growing wasabi in this environment would improve the commercial value of the wasabi crop.

Each of the wasabi-oriented food computer prototypes - entitled "Deterministic Agriculture Virtualization Environment", or DAVE - was constructed using accessible materials and techniques comparable to the PFC to promote replicability. DAVE was situated in the researcher's residential garage to facilitate ease of access over the duration of the experiment. Prior to the commencement of the experiments, each DAVE was outfitted with adolescent plants of Wasabia japonica (Misho variety) of an approximate age of 10 months sourced from a reliable commercial grower (Mountain View Wasabi, Surrey, BC, Canada). The experiment in each DAVE was allowed to run for 5-7 weeks to examine the impact of the DAVE environment. This time period was selected because 10-12 months of maturation is a more volatile period for both wasabi plant growth and AITC development [17], with the intent of seeing greater differences between test and control groups, thus producing more useful results.

Each DAVE prototype was designed according to the following paradigms:

1. DAVE mk1 (10 test plants, run time: Nov. 11, 2019 - Jan. 1, 2020) was designed around the existing literature. Following the conclusion of DAVE mk1's experiment, various qualitative results (plant health indicators, rhizome value indicators, etc.) were gathered. The results were stand-alone, presenting "absolute" results independent of any control group: the success of the prototype was based upon the success of the wasabi growth. The primary purpose of the $\mathrm{mk} 1$ prototype results was to be able to reverse-engineer the success or failure of various specific design ideas with the intent of making design improvements.

2. DAVE mk2 (5 test, 5 control plants, run time: Jan. 5, 2020 - Feb. 26, 2020) was designed following analysis of the results of DAVE mk1. Design was based upon that of DAVE mk1, with improvements reflecting the failures of the previous model. Over the course of the DAVE mk2 experiment, several general measurements were taken, including a time-lapse showcasing visual growth rate and plant health, as well as captures of the microclimate state via sensor data. At the terminus, quantitative results related to plant commercial quality (mass, AITC concentration) were collected and compared to a control group of plants of similar maturity and identical source and variety.

\section{DAVE mk1}

\section{Physical Construction and Hydroponics Materials}

The physical food computer was a rectangular prism (1 foot by 3-foot base, 3 feet tall) built out of corrugated plastic sheet, reinforced with plywood, and sealed with epoxy (see Figure 1A). The front face was hinged to allow for access to the plants. A deep-water-culture (DWC) hydroponics system, in which roots were totally submerged, was chosen due to both its similarity to wasabi's natural semi-aquatic environment [1], [18] and its ease of monitoring and controlling variables such as $\mathrm{pH}$, nutrient concentration, and water temperature. In addition, "HydroBall" pH-neutral porous hydroponics medium was used as the primary growth medium for root suspension and plant structural support because, as shown by Kangwon Province Agricultural Research \& Extension Services researchers Byeon et al., "Growth characteristics and yield [of wasabi] were higher in hydroball ... than granite [gravel] and river sand" [19]. The hydroponic basin was filled with hydroball, and the plants were then placed equidistant from one another inside 
the basin, with hydroball covering up to the top of the rhizome, and the water level calibrated to reach the top of the root system (see Figure 1B).
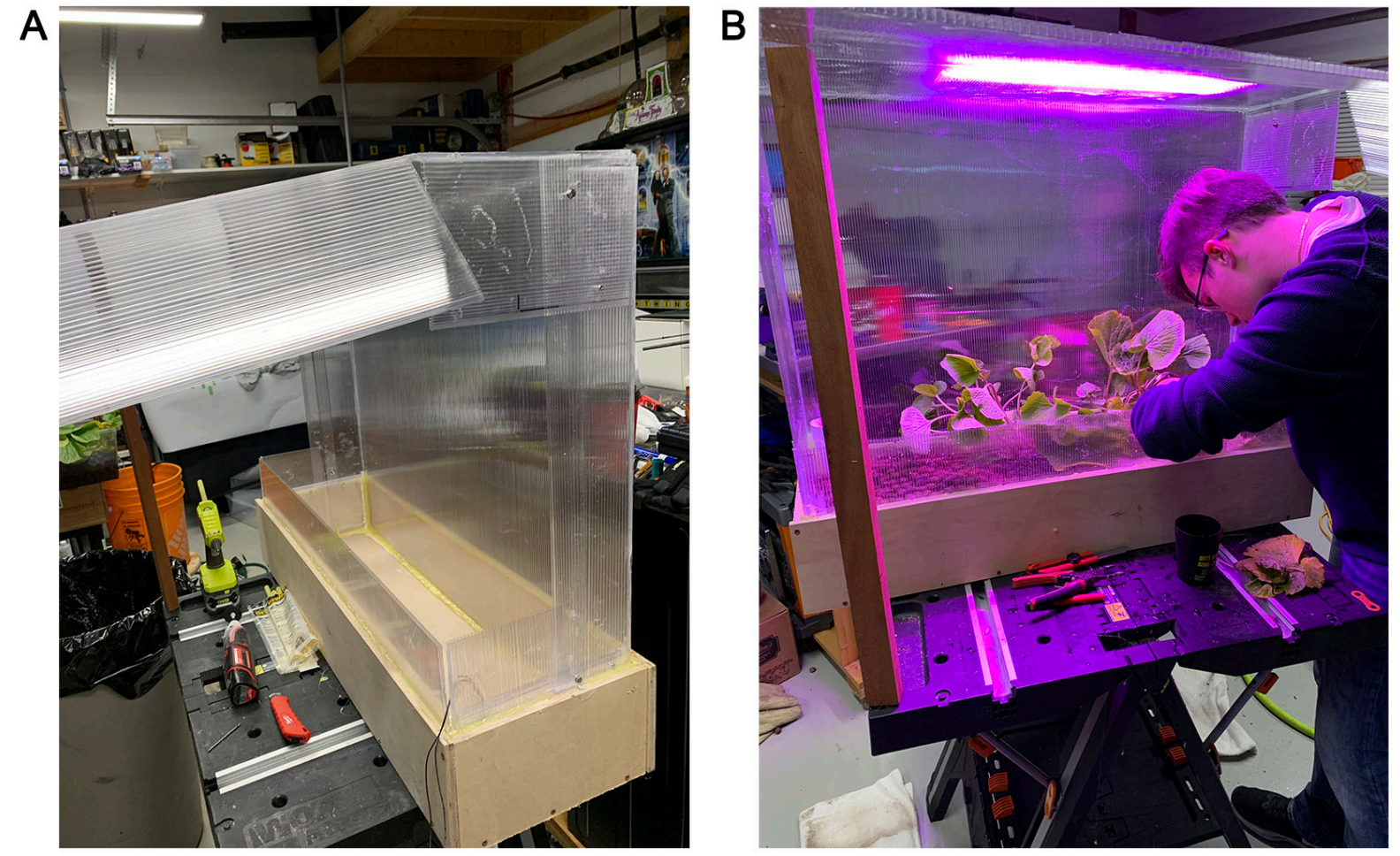

Figure 1. DAVE mk1. In 1A, hinged face open, showing sealant, corrugated plastic, and residential garage. In 1B, hydroball and plants are placed, and light is affixed.

\section{Automation Materials}

In order to address the computer automation component, a Raspberry Pi $3 \mathrm{~B}+$ was used as the main computer, mimicking the PFC. This allowed for native camera capture, local SQL database hosting, a large storage capacity, and native support for the implementation of the Python programming language for the main source code.

A variety of sensors were used to capture the state of the microclimate with regard to the variables in question. DAVE mk1 included a humidity and air temperature sensor (DHT22), a water temperature sensor (DS18B20), a water level float sensor, a Vernier pH sensor, and a Vernier EC sensor. The Vernier sensors were connected to an Arduino UNO rev3 via the SparkFun Vernier Interface Shield, and the data was transferred to the main computer via USB serial. The remainder were connected to the Raspberry Pi GPIO (General Purpose Input/Output) bus via solderless breadboard (see Figure 2). These sensor choices are similar to those included in the design of the PFC, with changes made only due to availability and cost.

In order to affect change on the microclimate, several actuators were installed. These include a $72 \mathrm{~W}$ thermoelectric air cooler/heater mechanism (which also functioned as the primary circulation fan), a $240 \mathrm{~W}$ thermoelectric water cooler block, a 32W LED horticulture lighting system, a circulation pump (always active), an oxygenation stone (always active), and a peristaltic water addition pump, all controlled by the Raspberry Pi via a relay board and powered using a $12 \mathrm{~V}$ power supply. 


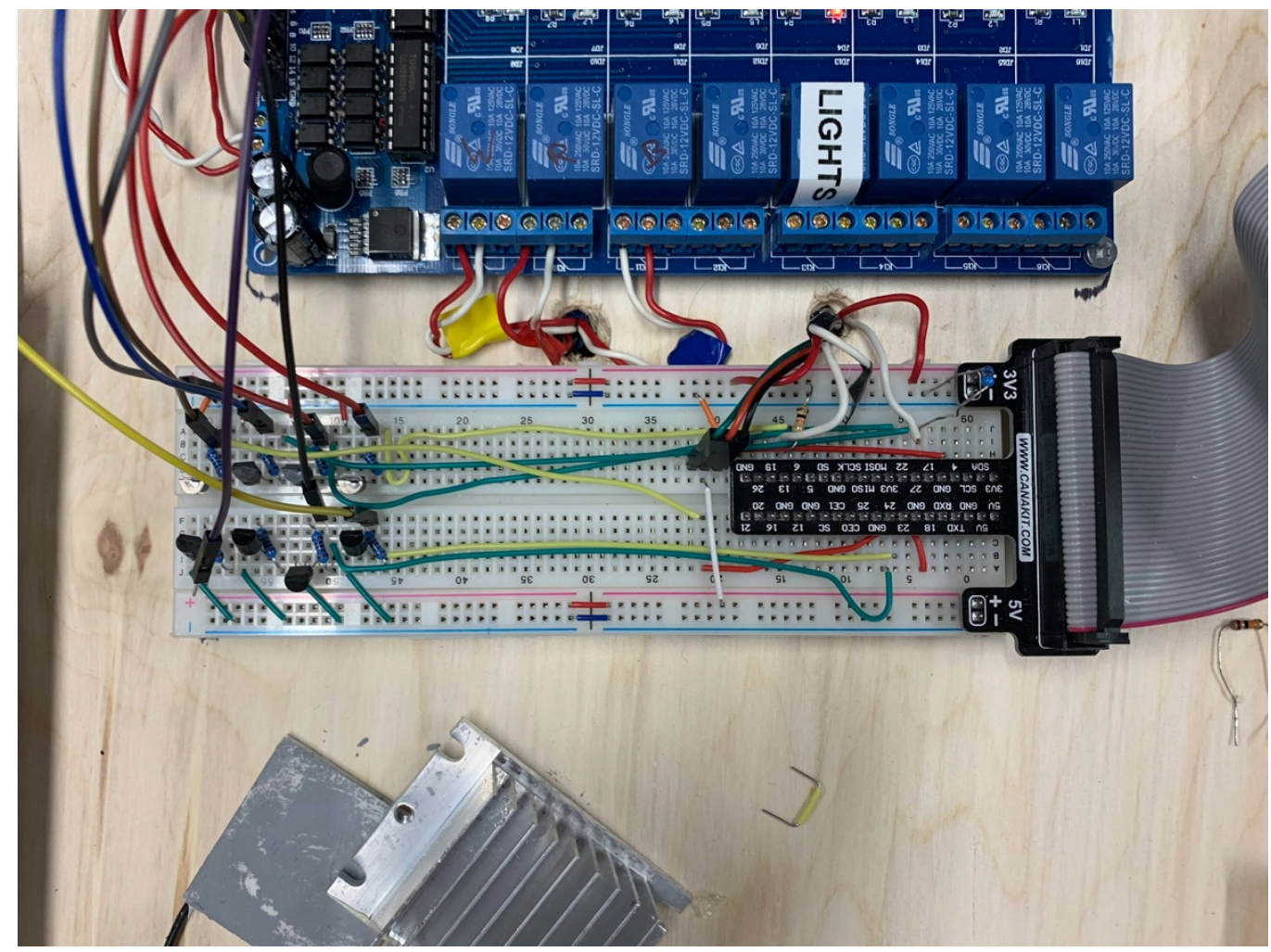

Figure 2. DAVE electronics. Shows Raspberry Pi GPIO connector (right) with various sensor connections, and transistor (left) amplified signal to relay board (top) controlling actuators.

\section{Automated Method}

The program executed by the Raspberry Pi, written in Python, follows the OpenAG model of "Sense, Plan, Act" [14]: the food computer maintains a state of "induced homeostasis" via sensing the current state, planning how to affect change in order to approach given optimal values, and then acting by implementing those changes. This runs perpetually and serves as the main DAVE program. The optimal environment variable values were collected from the examined literature, and were all induced in parallel:

1. Air temperature: $\mathbf{1 2}^{\circ} \mathbf{C}-15^{\circ} \mathbf{C}$ [9], sensed by DHT22 and induced by thermoelectric air cooler/heater.

2. Water temperature: $\mathbf{1 0}^{\circ} \mathbf{C}-13^{\circ} \mathbf{C}$ [9], sensed by DS18B20 and induced by water cooler (tended to increase due to air temperature being higher).

3. $\mathrm{pH}$ : 6.0-6.5 [1], sensed by Vernier $\mathrm{pH}$ probe, no actuator (set during water pre-mixing).

These values were maintained through induced homeostasis; when a value trended too low or too high, the related actuator was activated to counteract this trajectory. When the value re-entered the optimal range, the actuator was shut off. Various other components of the wasabi growth environment were controlled in addition to the variables listed, including water level in the basin (sensed by the water level float sensor and increased by peristaltic water addition pump), lighting (on a consistent activation schedule, 6 hours a day), and water oxygenation (maintained high via aeration stone). 


\section{Active Method}

Due to the nature of DAVE mk1 being a first prototype, not all design components could be automated. First and foremost, the water being added had to be premixed with the appropriate concentration of nutrients, $\mathrm{pH}$ balanced according to optimal values using typical hydroponic $\mathrm{pH}$ up and down solutions (General Hydroponics $\mathrm{pH}$ UP \& $\mathrm{pH}$ DOWN), and stored in a reservoir with the peristaltic addition pump's intake hose inside. In hydroponics, rather than being able to draw nutrients readily from nutrient-rich soil, all nutrients the plant requires must be found in solution. Therefore, a balanced two-part A+B nutrient programme (Dutch Nutrient Grow AB), along with a commonly-used calcium-magnesium-iron supplement (Botanicare CAL-MAG Plus), as directed on their respective bottles, should serve as an effective hydroponic equivalent to "slow release 12-12-12 fertilizer" already shown to be effective in traditional farming [1]. Executing the active method as needed, the automated method was allowed to run for the duration of the experiment. The plants were checked on multiple times a week without disturbing the environment, with any occurrences noted.

\section{Results and Analysis}

Nearly constant leaking out of the four bottom corners suggests unreliable assembly techniques. Root rot noted 5/12/2019: Putrid rotting smell and white sludge on roots (see Fig 4). Primarily attributed to any or all of poor water oxygenation, low circulation rate, low water refresh rate (therefore poor nutrient cycling), elevated water temperature, and/or not enough space between plants. Aphid infestation noted 1/1/2020: Significant and sudden negative effects on overall plant health (see Fig 5) including petiole flaccidity, severe leaf curl, and leaf chlorosis/death. Can be primarily attributed to air circulation problems and lack of insecticidal preparations. This occurrence, paired with a subsequent re-review of literature, suggests that wasabi is particularly susceptible to aphids. While, initially, the intended product of the DAVE mk1 experiment was chemical analysis of the wasabi crop, the sudden failure of the crop meant that this was not possible. Instead, an analysis of DAVE mk1's design was performed, identifying several key failures: a lack of time lapse and sensor data collection, issues with water leakage, poor air circulation and gas exchange, and improperly allocated space between plants. It can be concluded, however, that the most significant factor contributing to the plants' demise was the hydroponic method selected (DWC).
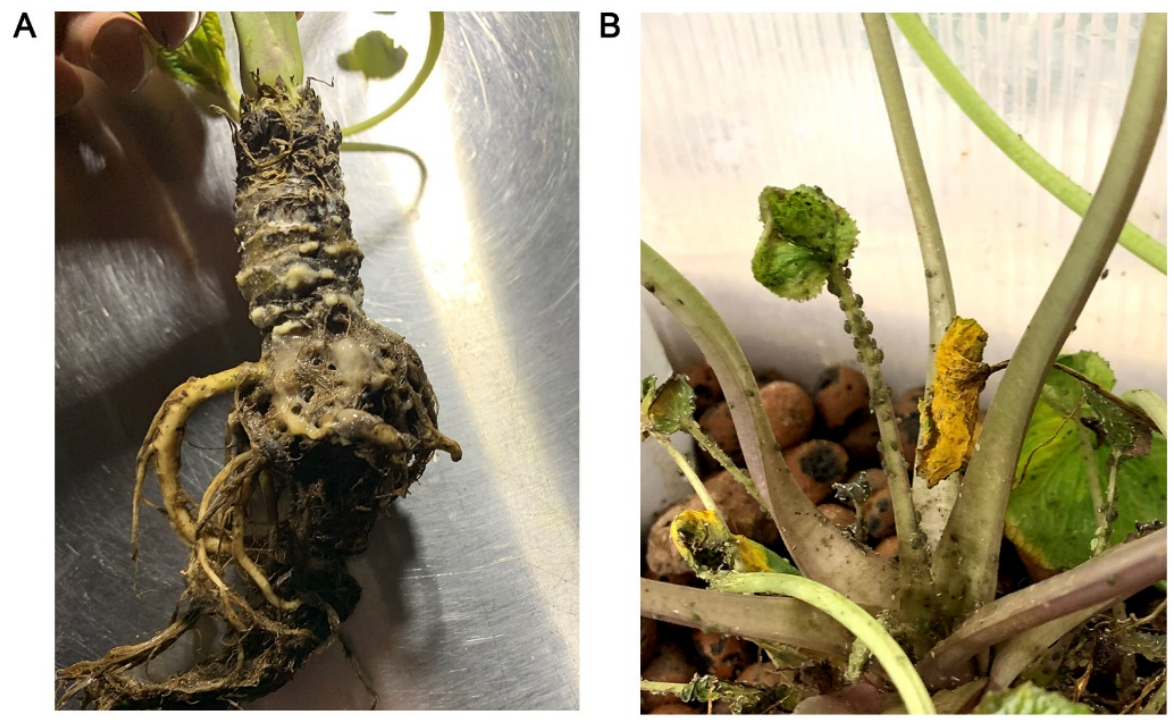

Figure 3. DAVE mk1 results. In 3A, root rot is shown. In 3B, aphid infestation is shown, along with resultant plant conditions. 


\section{DAVE mk2}

\section{Physical Construction and Hydroponics Materials}

The physical food computer consisted of a single piece 200L rugged plastic tote to avoid leaking. Walls were constructed from corrugated plastic sheets and reinforced with 1" PVC tubing. The front face was affixed with removable nuts to allow for access to the plants. The lid of the tote was used as a lid (see Figure 4A). The hydroponic basin was filled with hydroball. The plants were then placed equidistant from one another inside the basin, with hydroball covering up to the base of the rhizome (see Figure 4B).
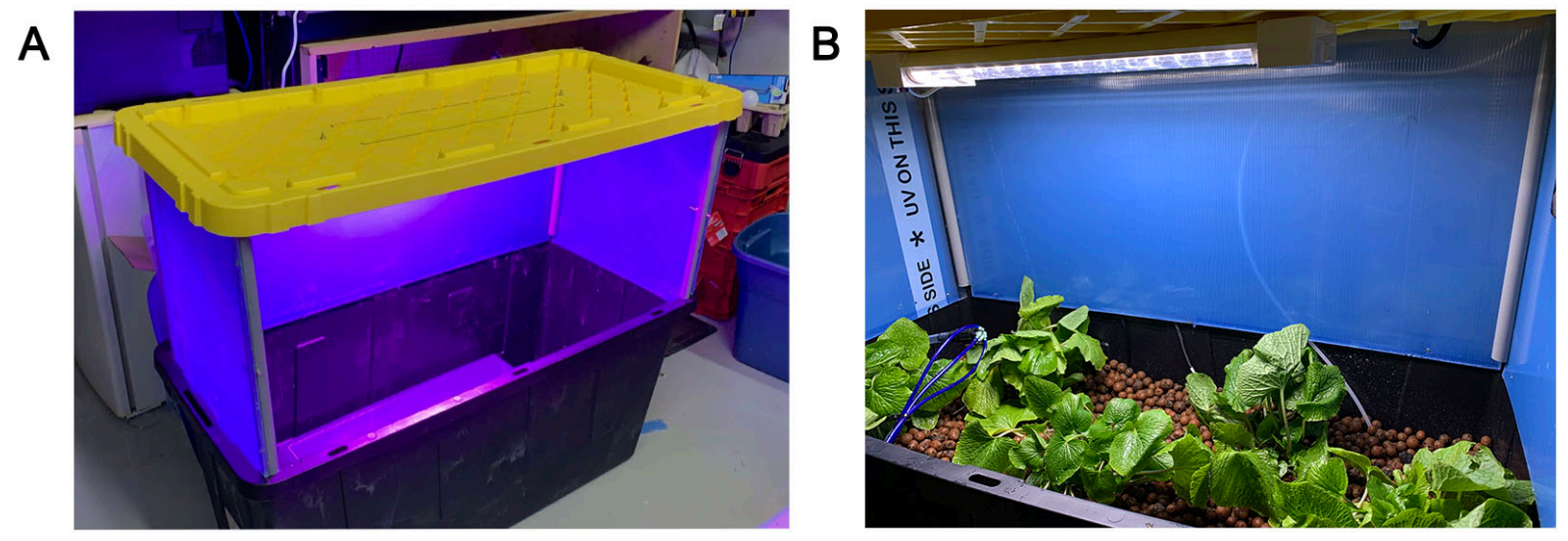

Figure 4. DAVE mk2. In 4A, the tote base and lid are shown, with corrugated plastic walls and supporting PVC tubing. In 4B, hydroball and plants are added, along with lighting, drip irrigation system, and various sensors.

A drip irrigation hydroponic technique where the base of each plant was indirectly sprayed with water continuously was chosen to rectify the failures observed in the results of DAVE mk1. Instead of filling the basin with water up to the root level, which could result in root rot, water was added only to cover the circulation pump intake and air stone.

The majority of non-construction materials (Arduino, Raspberry Pi, most sensors and actuators, circulation pump) were harvested upon disassembly of DAVE mk1 and re-used in mk2. Exhaust and intake fans were added to mitigate aphid problem and to more effectively control humidity. Air stone size was tripled to promote better oxygenation and prevent root rot.

\section{Automated Method}

Alongside the main protocol developed in DAVE mk1, two forms of live data collection were introduced to promote greater insight into the wasabi growth process, as well as to hold DAVE accountable. The data collected from the sensors was compiled and pushed to a local MySQL database every 15 seconds. Furthermore, a camera captured an image from a birds-eye-view every 20 minutes.

\section{Active Method}

The same nutrient solution preparation method was used for DAVE mk2, but was now supplemented with " $2 \%$ ammonium sulfate [spray, because use] one to three months before harvest [has been shown] to enhance flavor" [1]. Water reservoir refill only needed to be done weekly, as the leaking issue present in DAVE mk1 had been fixed, 
reducing the water consumption significantly. The daily routine consisted of spraying all leaves with water and removing leaves that were $>50 \%$ chlorotic, were waterlogged, had a very limp petiole, or had undergone severe physical damage. The weekly routine consisted of draining all water from the basin, refreshing the water supply bucket, filling to the standard fill line ( 60 liters), and spraying all leaves with $2 \%$ ammonium sulphate solution.

Once again, the automated method was allowed to run for the duration of the experiment, while the active method was executed as stated above. All non-invasive observations, however, were now recorded automatically through the sensor database and time-lapse footage, further reducing interference.

\section{Extraction Method}

At the conclusion of the experiment on February 26th, 2020, the control set was received. Five plants - identical variety, age, and source - were purchased in advance from the commercial grower (plants 6-10), in addition to the 5 plants of the test set (plants 1-5), but were left to grow in the standard commercial environment by the grower for use as a control set. All 10 plants were prepared for GCMS in a high school chemistry lab in a method widely used across the field [5], [8]-[12], [18]:

1. Each plant was washed with water to remove dirt, dust, etc.

2. Each plant was dissected into its 4 component tissue groups (leaves - LE, petioles - PE, rhizome - RH, and roots - RO), resulting in a total of 40 tissue samples ( 2 sets $x 5$ plants/set $x 4$ tissue groups/plant);

3. Each tissue sample's mass was recorded.

4. All tissue samples were stored in resealable plastic bags and deep-frozen overnight using a household freezer until internal temperature reached $<-18^{\circ} \mathrm{C}$.

5. All tissue samples were stored in a cooler with ice packs to preserve temperature.

The following was performed on all samples, in the order that maximised each samples' time spent in the cooler:

6. A sample was homogenized with $20 \mathrm{~mL}$ distilled water (to promote homogeneity) in a household food processor for 3 minutes to form a paste.

7. $0.6 \mathrm{~g}$ of the homogenized sample was added to a sterilized $1.5 \mathrm{~mL}$ microcentrifuge vial. The vial was labelled according to plant number and tissue (i.e. 9 RO for plant 9 , root tissues).

8. The vial was filled to $1.5 \mathrm{~mL}$ with light mineral oil, then incubated for one hour at room temperature $\left(20^{\circ} \mathrm{C}\right)$ with occasional agitation.

9. Extract (oil phase) was separated from solid matter and water phase via centrifugation $\left(5 \mathrm{~min} @ \sim 20^{\circ} \mathrm{C}, \sim 12000 \mathrm{~g}\right)$.

10. Oil phase (containing AITC) was pipetted from the now extracted sample into a new sterilized and labelled $1.5 \mathrm{~mL}$ microcentrifuge vial.

11. The extracted sample was returned to the cooler for storage.

Once all tissue samples were processed, the extracted samples were stored in a household fridge $\left(\sim 3^{\circ} \mathrm{C}\right)$ overnight.

\section{GCMS Method}

The following was performed on 16 samples at a time (all 4 samples from 4 plants), keeping the remainder in a cooler with ice packs, and executed in a university chemistry lab with direction and assistance from a chemistry professor and lab technician:

1. $0.5 \mathrm{~mL}$ of sample was partitioned against $0.5 \mathrm{~mL}$ methanol using a precision pipette in a $1 \mathrm{~mL} \mathrm{GC}$ vial, and agitated consistently for $2 \mathrm{~min} @ 20^{\circ} \mathrm{C}$. 
2. The samples were lined up in the autoinjector of an Agilent Technologies 7890B GC system fitted with an Agilent Technologies HP5MS column (30m, 0.25mm ID, 0.25micron stationary/solid phase);

3. The GC system was set to splitless mode with a $1 \mu \mathrm{L}$ injection volume taken $8.6 \mathrm{~mm}$ off bottom of vial (to only take up the methanol phase containing AITC), transfer line $250^{\circ} \mathrm{C}$, inlet port $250^{\circ} \mathrm{C}$.

A "general sweep" primary test run was conducted using sample 9RO to determine the optimal GC settings for AITC analysis (start $40^{\circ} \mathrm{C}$, end $325^{\circ} \mathrm{C}$, rate $10^{\circ} \mathrm{C} /$ minute, 2 minute solvent delay, MS scan from 30amu to 500amu). This took 28.5 minutes. The AITC peak was detected (see Figure 5) and the GC settings were optimized to isolate that peak and increase efficiency $\left(\mathrm{GC}\right.$ range changed to start $40^{\circ} \mathrm{C}$, end $120^{\circ} \mathrm{C}$, rate $20^{\circ} \mathrm{C} /$ minute).

Samples were processed independently by the lab technician in accordance with lab availability, which was totally out of the control of the researcher. Scheduling ended up being:

1. Plants 3, 5, 6, and 8: Run 1, Feb. 28, 2020

2. Plants 1, 2, 4, and 7: Run 2, Mar. 6, 2020

3. Plants 9 and 10: Run 3, Mar. 9, 2020

Following Run 3, the results were sent to the researcher for analysis.

\section{Results and Analysis}

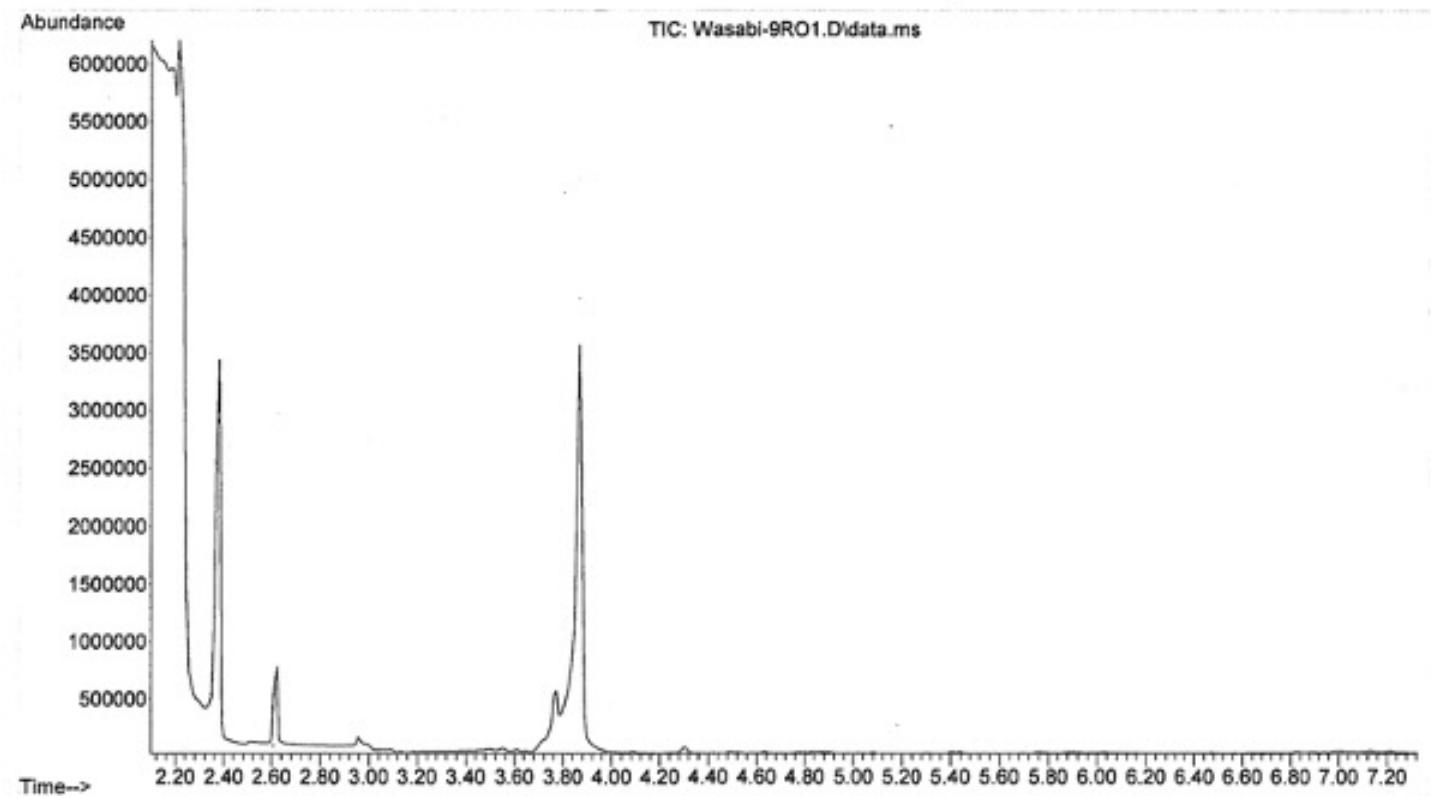

Figure 5. GCMS "general sweep" results. Peaks indicate high abundances of particles exiting at a given time. All GCMS data was in this format.

The peaks in Figure 5 represent the various compounds in the sample, being detected by the MS across time as they exit the GC column. In order to determine the abundance of AITC, we must first locate it in the chromatography stack. The peak at time 3.867 is further examined in Figure 6. 

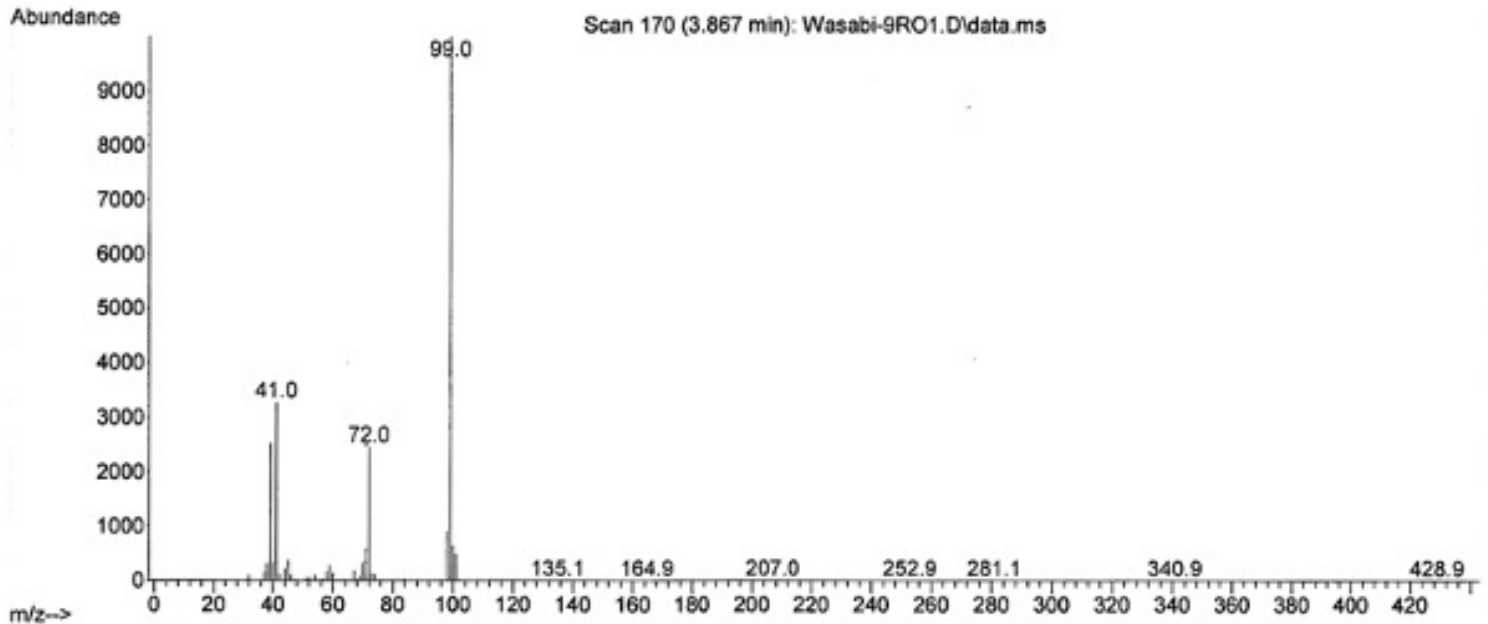

Figure 6. MS graph of sample $9 \mathrm{RO}$ at time 3.867. Shows the abundance of various charged particles entering the MS from the GC column.

This peak was then indexed and compared against the NIST MS database, where potential matches were ranked according to their accuracy. AITC's standard MS data (see Figure 7) matches the MS data in Figure 6 with $\mathbf{8 7 \%}$ accuracy, indicating a highly probable match.

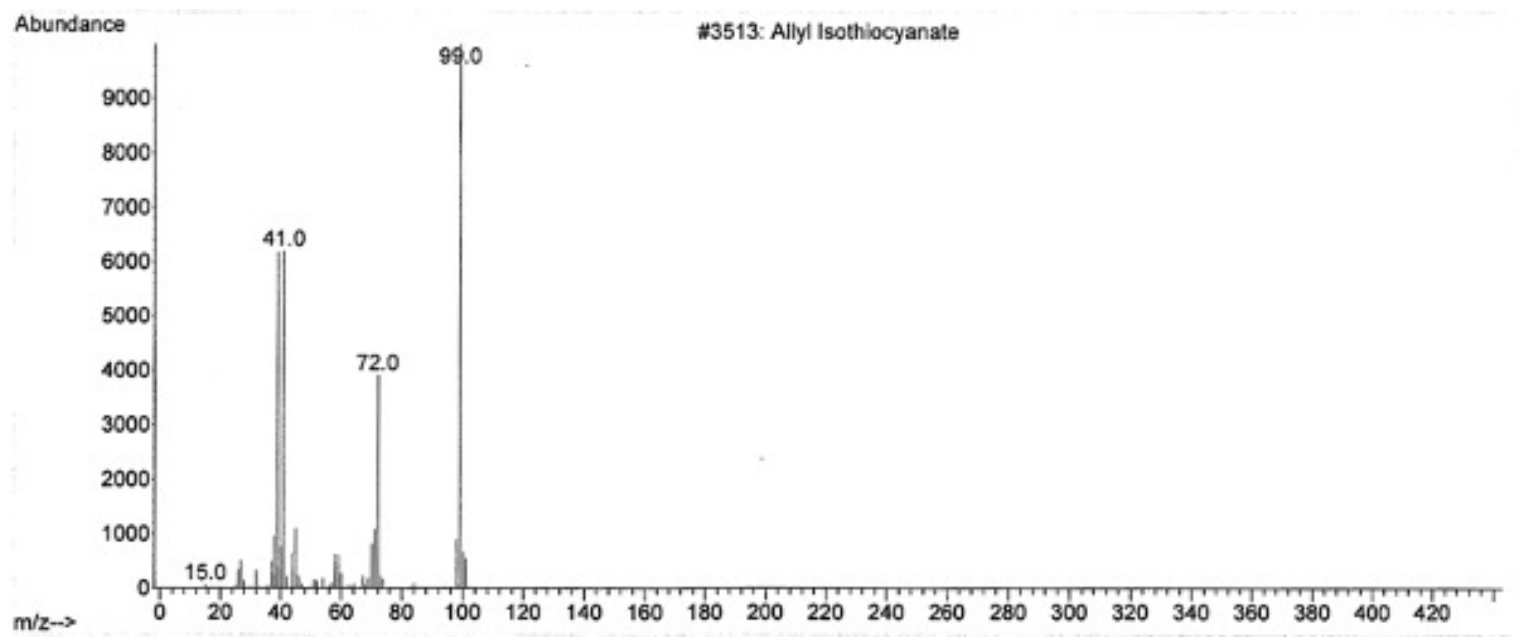

Figure 7. Standard MS graph of AITC. Taken from NIST MS database; matches data in Figure 6 with $87 \%$ accuracy.

The compounds of interest in terms of wasabi quality - all ITCs to some degree, but primarily AITC - are exhibited in surprising clarity and precision through the GCMS process. The peaks classified as AITC are integrated across time from peak start to finish to determine the total abundance of the compound. This abundance is proportionate to the concentration of the compound in the tissue sample.

Though we cannot determine the absolute concentration (typically expressed in $\mathrm{ppm}$ or $\mathrm{mg} / \mathrm{kg}$ ), we can compare the abundances of AITC in each of the sample groups. Given that all plant tissues from both control and experimental set underwent identical processes, we can determine a numerical coefficient representative of the relative efficacy of DAVE mk2 as a growth environment as compared to a commercial standard growth environment. 
Table 1. Integrated AITC abundance averages in and the ratio between test set samples and control set samples. Overall ratio: $\mathbf{1 6 9 . 3 \%}$.

\begin{tabular}{|c|c|c|c|c|}
\hline Plant & Leaves & Petioles & Rhizome & Roots \\
\hline Control Set & $180,852,304$ & $110,243,350$ & $459,942,347$ & $54,253,557$ \\
\hline Test Set & $168,462,643$ & $183,569,305$ & $600,866,947$ & $155,637,973$ \\
\hline Abundance Ratio & $93.1 \%$ & $166.5 \%$ & $130.6 \%$ & $286.9 \%$ \\
\hline
\end{tabular}

Mass data gathered prior to the extraction phase of the method was also examined.

Table 2. Average mass ratio between test set tissue samples and control set tissue samples, per organ.

\begin{tabular}{|c|c|}
\hline Plant Organ & Mass Ratio \\
\hline Leaves & $12.2 \%$ \\
\hline Petioles & $22.1 \%$ \\
\hline Rhizome & $64.1 \%$ \\
\hline Roots & $41.2 \%$ \\
\hline Overall & $\mathbf{2 5 . 3 \%}$ \\
\hline
\end{tabular}

Certain limitations to analysis present themselves upon further inspection of the data. For example, a gradual yet significant drop in AITC abundance was observed across samples as their total storage time increased. This drop was originally indicated to the researcher by the lab technician, and was shown to be consistent across all samples, regardless of group, and indicative of a chemical breakdown over time. Through conferencing with both the lab technician and chemistry professor, it was determined that only samples analyzed on the same date were comparable. This type of statistical analysis is called blocking.

Furthermore, samples analyzed during GCMS run 2 were missing key data points and were full of outliers. This can be attributed to both human error and chemical breakdown at different rates. Also, samples analyzed during GCMS run 3 were only of the control group, so no blocked analysis within the run can be performed, as there is nothing to compare these samples to. These circumstances render the data from these both of these runs particularly useless. There is precedent in this field, however, for using small samples sizes of virtually identical wasabi plants to represent a larger population [15]. Of the useable data gathered only during GCMS run 1, a trend can be observed.

\section{Conclusion}

Although the concentration of AITC in DAVE mk2 plants is $69.3 \%$ higher on average than those grown in a commercial environment (see Table 1), the total mass is lower in the test set than in the control set across all plant organs, with a $74.7 \%$ decrease overall (see Table 2 ).

These results points to two possible conclusions, depending on the specific optimization focus:

1. The overall yield is lower, resulting in a lower commercial value, meaning that DAVE mk2 is ineffective if mass is the specific focus.

2. The negative relationship between AITC concentration and plant mass might be explained by the "dilution effect", in reverse. Therefore, if the goal is solely to increase concentration of AITC, with no specific attention paid to yield, DAVE mk2 is highly effective.

\section{Limitations}

1. There is little information publicly available about growing wasabi hydroponically in artificial/commercial environments. Much knowledge is proprietary and owned by vendors unwilling/unable to cooperate with researchers. 
2. The ages of the plants transplanted into DAVE are of indeterminate accuracy, which is understandable, given the nature of commercial wasabi agriculture.

3. The PFC, though open source, has incomplete documentation to the point where the creators have said on their public forums not to attempt to replicate the PFC. Thus, the use of the PFC as-is was impossible, and an analogue was designed based on the available PFC documentation.

4. During the chemical analysis process, better homogenization, more rapid GCMS, and multiple tests of the same sample could have all been done, and would have resulted in more accurate data and better statistical analysis.

5. There exists no prior research on the Misho variety of Wasabia japonica.

\section{Implications and Future Research}

DAVE marks the first collision between cyber-agriculture and the world of wasabi, and it is believed that "Computercontrolled growth environments are a promising approach for the future of agriculture, potentially maximizing production and quality and minimizing waste and cost" [15]. In an ever-evolving marketplace, cyber-agriculture could be leveraged to maximise cultural impact through flavour, and as a means of making ingredients more accessible.

Should the DAVE prototypes continue to be developed, several design components could be improved:

1. Better/more sensors, to more accurately capture the growth environment.

2. A longer experiment duration, perhaps from seedling to typical commercial maturity would yield results of greater significance.

3. Detailed open-source documentation and the use of more widely available "maker-style" construction techniques (i.e. 3D printing, etc.) would facilitate increased repeatability. Fellow researchers could easily implement a DAVE instance in their own lab and repeat this experiment.

\section{Acknowledgements}

Thanks are given to the following individuals: D. Hickie and Dr. V. Pontieri - For providing highschool chemistry lab space and access to certain materials and tools. Dr. S. Wylie - For assisting during GCMS analysis and facilitating access to university chemistry lab. S. Straughan - For steadfast support and guidance, and a dose of healthy skepticism.

\section{References}

1. C. I. Chadwick, T. A. Lumpkin, and L. R. Elberson, "The Botany, Uses and Production of Wasabia japonica (Miq.) (Cruciferae) Matsum," in Econ. Bot., vol. 47, no. 2, pp. 113-135, Apr.-Jun. 1993, doi: 10.1002/mnfr.201700990.

2. J. A. Douglas and J. M. Follett, "Initial research on the production of water-grown wasabi in the Waikato," in Proc. Agron. Soc. New Zealand, 1992, vol. 22, pp. 57-60. [Online]. Available: https://www.agronomysociety.org.nz/files/1992_11._Water-grown_wasabi_prod_in_Waikato.pdf

3. R. J. Martin and B. Deo, "Preliminary assessment of the performance of soil-grown wasabi (Wasabia japonica (Miq.) Matsum.) in New Zealand conditions," in New Zealand J. Crop and Hort. Sci., vol. 28, no. 1, pp. 45-51, 2001, doi: 10.1080/01140671.2000.9514121. 
4. A. Gałczyńska, P. Trzcinska, M. Gumienna, K. Nowak, and R. Hołubowicz, "Production of Japanese Horseradish (Wasabia japonica (Miq.) Matsumara) in Poland. Chemical Contents of Roots," in Notulae Botanicae Horti Agrobotanici Cluj-Napoca, vol. 45, no. 2, pp. 466-472, 2017, doi: 10.15835/nbha45210887.

5. T. Sultana, G. P. Savage, D. L. McNeil, N. G. Porter, and R. J. Martin, "Flavour components in the rhizome of soil grown wasabi," in Proc. Nutr. Soc. New Zealand, Nov. 2000, vol. 25, pp. 95-106. [Online]. Available: https://www.researchgate.net/profile/Geoffrey_Savage/publication/263581871_Flavour_components_in_the_rhizome_of_soil-grown_wasabi/links/0f31753b4d1bd54bae000000/Flavour-components-in-therhizome-of-soil-grown-wasabi.pdf

6. T. Sultana and G. P. Savage, "Wasabi - Japanese Horseradish," in Bangladesh J. Sci. and Ind. Research, vol. 43, no. 4, pp. 443-448, 2008, doi: 10.3329/bjsir.v43i4.2234.

7. L. Bell, O. O. Oloyede, S. Lignou, C. Wagstaff, and L. Methven, "Taste and Flavor Perceptions of Glucosinolates, Isothiocyanates, and Related Compounds," in Mol. Nutr. and Food Res., vol. 62, no. 18, Sept. 2018, doi: 10.1002/mnfr.201700990.

8. S. W. Shin et al., "Investigation Of Total Phenolic, Total Flavonoid, Antioxidantand Allyl Isothiocyanate Content In The Different Organs Of Wasabi Japonica Grown In An Organic System," in Afr. J. Trad., Complem. and Alt. Medicines, vol. 11, no. 3, pp. 38-45, 2014, doi: 10.4314/ajtcam.v11i3.7.

9. S. Oguni, K. Kakibuchi, and Y. Katayama, "Effects of Environmental Controls on the Growth of Wasabi (Eutrema japonica (Miq.) Koidz.) in a Nutrient Solution Cultivation System," in Environ. Contr. Biol., vol. 43, no. 3, pp. 181-191, 2005, doi: 10.2525/ecb.43.181.

10. T. Sultana, G. P. Savage, D. L. McNeil, N. G. Porter, R. J. Martin, and B. Deo, "Effects of fertilisation on the allyl isothiocyanate profile of above-ground tissues of New Zealand-grown wasabi," in J. Sci. Food and Agri., vol. 82, no. 13, pp. 1477-1482, Oct. 2002, doi: 10.1002/jsfa.1218.

11. R. J. Martin, B. Deo, and J. Depree, "Wasabi yield and isothiocyanate responses to fertiliser type," in Agron. New Zealand, vol. 32, pp. 121-125, 2002. [Online]. Available: https://www.agronomysociety.org.nz/files/2002_15._Wasabi_yield_isothiocyanate_response.pdf

12. R. A. Craigie, "Yield and quality response of wasabi (Wasabia japonica (Miq.) Matsumara) to nitrogen and sulphur fertilisers," M.S. thesis, Hort. Sci., Lincoln Univ., Canterbury, New Zealand. [Online]. Available: http://researcharchive.lincoln.ac.nz/handle/10182/2795

13. S. Tembe, S. Khan, and R. Acharekar, "IoT based Automated Hydroponics System," in Int. J. Sci. \& Eng. Res., vol. 9, no. 2, pp. 67-68, 2018. [Online]. Available: https://pdfs.semanticscholar.org/da9a/6233b72a23a9290152d79f3a9eb89b6089dd.pdf

14. C. Harper and M. Siller, "OpenAG: A Globally Distributed Network of Food Computing," in IEEE Pervasive Comput., vol. 14, no. 4, pp. 24-27, Oct.-Dec. 2015, doi: 10.1109/MPRV.2015.72.

15. A. J. Johnson, E. Myerson, J. de la Parra, T. L. Savas, R. Miikkulainen, and C. B. Harper, "Flavor-cyber-agriculture: Optimization of plant metabolites in an open-source control environment through surrogate modeling," in PLOS, Apr. 2019, doi: 10.1371/journal.pone.0213918. 
16. E. Ferrer et al., "Personal Food Computer: A new device for controlled-environment agriculture," presented at Future Technologies Conference, 2017, doi: 1721.1/110010.

17. H. S. Byeon, J. S. Seo, S. J. Lim, S. J. Heo, and S. M. Seo, "Changes of Allylisothiocyanate Content and Hardness of Rhizome by Months after Planting in Wasabia Japonica Matsum.," in Korean J. Medicinal Crop Sci., vol. 11, no. 3, pp. 186-189, 2003. [Online]. Available: https://www.koreascience.or.kr/article/JAKO200303042351927.pdf

18. T. Sultana, N. G. Porter, G. P. Savage, and D. L. McNeil, "Comparison of Isothiocyanate Yield from Wasabi Rhizome Tissues Grown in Soil or Water," in J. Agri. and Food Chem., vol. 51, no. 12, pp. 3596-3591, May 2003, doi: 10.1021/jf021116c.

19. H. S. Byeon et al., "Effects of Soil Physical properties on Growth in Wasabia japonica Matsum," in Korean J. Medicinal Crop Sci., vol. 9, no. 1, pp. 76-82, 2001. [Online]. Available: https://www.koreascience.or.kr/article/JAKO200103042338709.pdf 